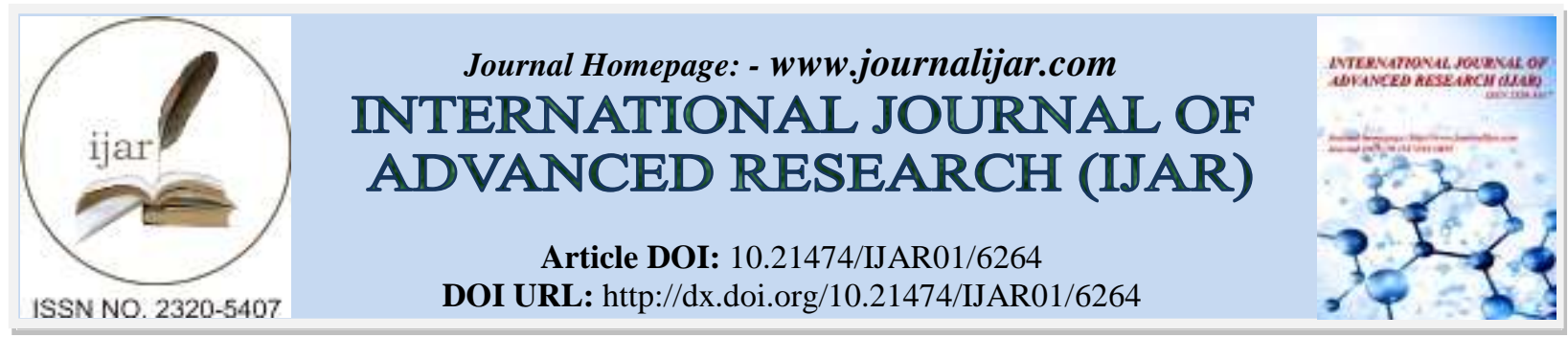

RESEARCH ARTICLE

\title{
CASE STUDY: BARIATRIC SURGERY BOON FOR MORBID OBESITY.
}

Supriya.

Registered Dietician Intern, Apollo Hospital, Bengaluru, Karnataka.

\section{Manuscript Info}

Manuscript History

Received: 10 November 2017

Final Accepted: 12 December 2017

Published: January 2018

Keywords:-

Extreme obesity, bariatric surgery.

\begin{abstract}
Bariatric surgery is a recognized and accepted approach for addressing weight loss and health conditions that occur as a result of morbid or severe obesity. Bariatric surgery is a generic term for weight loss surgery and is the best and effective method for enduring weight loss combined with change in lifestyle as well as diet. Lifestyle changes, dietary modifications, and regular exercise are required for optimal and lasting surgical weight loss. Successful outcomes after bariatric surgery require a multidisciplinary approach and extensive patient counseling, especially to diet and change in lifestyle with exercise. Experience suggests that medical therapies for weight loss (eg, dietary therapy, physical activity, behavior therapy) do not produce significant or longterm weight loss and that bariatric surgery together with dietary and lifestyle changes does. Bariatric surgery has been shown to be the most effective and durable treatment for morbid obesity. Patients undergoing bariatric surgery must commit to a program of lifestyle changes, diet, vitamin supplementation, with proper routine follow-up.
\end{abstract}

Copy Right, IJAR, 2018,. All rights reserved.

\section{Introduction:-}

According to national statistics, more than one-third of adults are obese. This prevalence of obesity has been described as an epidemic. Obesity contributes to medical comorbidities such as hypertension, diabetes, coronary artery disease, dyslipidemia, hyper triglyceridemia, obstructive sleep apnea, and osteoarthritis. Bariatric surgery is a recognized and accepted approach for addressing weight loss and health conditions that occur as a result of these classes of obesity. Bariatric surgical interventions cause weight loss by restricting the amount of food the stomach can hold, causing malabsorption of nutrients, or by a combination of both gastric restriction and malabsorption that results in hormonal changes.4 Surgeons, bariatricians, nurses, dieticians, psychologists, and physical trainers comprise the health care team that provides care to patients undergoing bariatric surgery. Most bariatric surgery centers recommend a multidisciplinary approach to sustained weight loss after bariatric surgery, which requires sustained dietary changes, lifestyle modification, and regular exercise.

Bariatric surgery is the term for operations to help promote weight loss.

There are three types of bariatric surgery:

1. LAP- BAND® system

2. Vertical Banded Gastroplasty or sleeve gastrectomy

3. Roux-en-Y Gastric Bypass 


\section{Lap - Band® System:-}

An adjustable silicone elastic band is placed around the upper part of the stomach creating a small pouch and restricting the passage of food.

\section{Gastric Bypass:-}

Gastric Bypass is the most frequently performed weight loss procedure in the United States. During this surgery, the upper stomach is stapled creating a small pouch that is completely divided from the remainder of the stomach.

\section{Vertical Banded Gastroplasty (Vbg):-}

VBG is a purely restrictive procedure in which the upper stomach is stapled and divided, forming a small pouch that reduces the size of the stomach and the amount of food the stomach can hold.

\section{Criteria for Bariatric surgery:-}

1. BMI of $40+$

2. Diabetes, heart disease or severe apnea

3. Related physical problems like physical mobility or physical function

\section{Laparoscopic Sleeve Gastrectomy:-}

1. Sleeve gastrectomy, or gastric sleeve, is a surgical weight-loss procedure in which the stomach is reduced to about $15 \%$ of its original size, by surgical removal of a large portion of the stomach, following the major curve.

2. The open edges are then attached together (typically with surgical staples, sutures, or both) to leave the stomach shaped more like a tube, or a sleeve, with a banana shape.

3. The procedure permanently reduces the size of the stomach. The procedure is performed laparoscopically and is not reversible.

\section{Potential side effects of bariatric surgery include, but are not limited to:-}

1. Nausea and vomiting

2. Gas and bloating

3. Lactose intolerance

4. Temporary hair thinning

5. Nausea and vomiting may occur, particularly in the first few days after surgery-vomiting is also common if you eat too quickly, or eat too much.

\section{Advantages:-}

1. The amount of food one can eat is restricted.

2. You are likely to feel fuller quicker and stay fuller for longer.

3. Weight loss starts from the time of surgery.

4. Weight loss tends to be faster than following the gastric band.

5. You can lose average 50\%-60\% of your excess weight.

6. As with the band, intestines remain intact so food is digested and absorbed as normal.

\section{Diet after gastric sleeve surgery:-}

1. 60-80 grams of protein a day

2. Eat protein first at all your meals

3. Eat slowly and chew well

4. Meals should take 40- 45 minutes

5. Avoid snacking on high calorie foods in between meals

6. Beverages less than 10 calories per serving

7. Vitamin/mineral supplementation for life as per doctors advice

8. Multivitamin (one a day, with food; not with calcium or dairy)

9. Calcium 1500-2000 mgs a day in divided doses at least 3 times a day and no more than $600 \mathrm{mgs}$ at one time, and apart from multivitamin and iron.

\section{Exercise:-}

1. Aim for at least 30 minutes on most days of the week, 5 minutes at a time, and gradually build up, as tolerated

2. Incorporate light weights about 2 months after surgery, and/or as your surgeon recommends 
3. Consider stretching and the importance of stress management program in your regimen

Case Presentation:-

A 41 years old male patient presented with morbid obesity. Patient has tried physical exercise and change in diet but there was no change in obesity. Admitted for further evaluation and bariatric surgery. Family history was nothing significant. Patient was known case of hypertension since 8 yrs and sleep apnoea. At the time of admission patient was acute febrile, his blood pressure was $130 / 80 \mathrm{~mm} / \mathrm{Hg}$ and pulse was 86 beats/min. Patient food habbit nonvegetarian. Patient has tried physical exercise, diet but there was no change. Patient has a social history of consuming tobacco (quit 2009), smoking (quit 2009). At present occasional alcohol consumption. His thirst, appetite and bowel normal.

Medication History:-

\begin{tabular}{|l|l|l|l|}
\hline MEDICINE & DOSE & FREQUENCY & PURPOSE \\
\hline Inj Supacef IV & $1.5 \mathrm{gm}$ & BD in 100 ml NS & Antibiotic \\
\hline Inj Pantadoc IV & $40 \mathrm{mg}$ & OD & Antacid \\
\hline Inj Emset IV & $4 \mathrm{mg}$ & SOS & To stop vomiting \\
\hline Inj Voveran IV & $75 \mathrm{mg}$ & BD & Pain killer \\
\hline Duolin NEB & $2.5 \mathrm{ml}$ & TDS & Bronchodialator \\
\hline Inj Clexane & $49 \mathrm{mg}$ & OD & Anticoagulant \\
\hline T. Amlong & $5 \mathrm{mg}$ & $1-0-1$ & Anti - hypertensive \\
\hline T. Olemzest & $40 \mathrm{mg}$ & $1-0-0$ & Anti- hypertensive \\
\hline T. Olmezest & $20 \mathrm{mg}$ & $0-0-1$ & Anti - hypertensive \\
\hline T. Carvedicol & $6.2 \mathrm{mg}$ & $1-0-1$ & Anti - hypertensive \\
\hline
\end{tabular}

Surgery: Laparoscopic Sleeve Gastrectomy procedure done on $2^{\text {nd }}$ day

Anthropometric Data:-

$\begin{array}{ll}\text { Height } & -171 \mathrm{~cm} \\ \text { Current body weight } & -130 \mathrm{~kg} \\ \text { Ideal body weight } & -71 \mathrm{~kg} \\ \text { Body mass index } & -44.45 \mathrm{~kg} / \mathrm{m}^{2}\end{array}$

Biochemical Parameters:-

\begin{tabular}{|l|l|l|}
\hline PARAMETER & RESULT & NORMAL RANGE \\
\hline Haemoglobin & $14.7 \mathrm{gm} / \mathrm{dl} *$ & $11.5-14.5$ \\
\hline WBC & 8.3 thousands/cumm & $4-11$ \\
\hline RBC & 5.2 millions/cmm & $4.5-5.5$ \\
\hline Platelet count & 222 thousands/cumm & $150-450$ \\
\hline Polymorph & $52 \%$ & $40-75$ \\
\hline Lymphocyte & $32 \%$ & $20-45$ \\
\hline Monocyte & $9 \%$ & $2-10$ \\
\hline Eosinophil & $6 \%$ & $1-6$ \\
\hline Basophil & $1 \%$ & $0-1$ \\
\hline Packed cell volume & $44 \%$ & $36-46$ \\
\hline Serum alkaline phosphatase & $46 \mathrm{U} / \mathrm{L}$ & $30-120$ \\
\hline Serum bilirubin total & $0.4 .6 \mathrm{mg} / \mathrm{dl}$ & $0.2-1.2$ \\
\hline S. bilirubin direct & $0.09 \mathrm{mg} / \mathrm{dl}$ & $0.0-0.4$ \\
\hline S. bilirubin indirect & $0.01 \mathrm{mg} / \mathrm{dl}$ & $0.1-1.0$ \\
\hline S. Total protein & $7.7 \mathrm{~g} / \mathrm{dl}$ & $6.0-8.5$ \\
\hline S. Albumin & $4.5 \mathrm{~g} / \mathrm{dl}$ & $3.5-52$ \\
\hline S. Globulin & $3.2 \mathrm{~g} / \mathrm{dl}$ & $2.0-4.0$ \\
\hline A/G Ratio & 1.4 & $0.8-2.0$ \\
\hline S. SGPT & $63 * \mathrm{U} / \mathrm{L}$ & $5-40$ \\
\hline S. SGOT & $26 \mathrm{U} / \mathrm{L}$ & $5-45$ \\
\hline S. Gamma GT & $48 * \mathrm{U} / \mathrm{L}$ & $10-45$ \\
\hline
\end{tabular}




\begin{tabular}{|l|l|l|}
\hline S. Urea & $19 \mathrm{mg} / \mathrm{dl}$ & $15-45$ \\
\hline S. Creatinine & 0.81 & $0.81-1.44$ \\
\hline S. Sodium & $139 \mathrm{mmol} / \mathrm{L}$ & $135-145$ \\
\hline S. Potassium & $3.8 \mathrm{mmol} / \mathrm{L}$ & $3.5-5.5$ \\
\hline S. Bicarbonate & $21^{*}$ & $22-29$ \\
\hline Plasma glucose & $92 \mathrm{mg} / \mathrm{dl}$ & $70-140$ \\
\hline T3 & $3.64 \mathrm{pg} / \mathrm{ml}$ & $2.5-3.9$ \\
\hline T4 & $0.87 \mathrm{ng} / \mathrm{dl}$ & $0.61-1.12$ \\
\hline TS4 & $4.34 \mathrm{IU} / \mathrm{ml}$ & $0.34-5.6$ \\
\hline
\end{tabular}

24 Hour Dietary Recall:-

\begin{tabular}{|c|c|c|c|c|}
\hline+2 & Meal & Menu & & Amount \\
\hline 7:00 am & Early Morning & Tea & & $100 \mathrm{ml}$ \\
\hline \multirow[t]{3}{*}{ 9:00 am } & \multirow[t]{3}{*}{ Breakfast } & Dosa & & $3 \mathrm{Nos}$ \\
\hline & & Sambar & & 1 cup \\
\hline & & Chutney & & $1 / 2$ cup \\
\hline 11:00 am & Mid Morning & Tea & & $100 \mathrm{ml}$ \\
\hline \multirow[t]{3}{*}{$1: 30 \mathrm{pm}$} & \multirow[t]{3}{*}{ Lunch } & Rice & & 1 cup \\
\hline & & Fish Curry & & 1 cup \\
\hline & & Veg Salad & & 1 cup \\
\hline 4:30 pm & Evening Snack & Tea & & $100 \mathrm{ml}$ \\
\hline \multirow[t]{2}{*}{ 9:00 pm } & \multirow[t]{2}{*}{ Dinner } & Chapathi & & 4 Nos \\
\hline & & Chicken Curry & & 1 cup \\
\hline & Energy (Kcal) & Protein (gm) & Fat (gm) & CHO (gm) \\
\hline Requirements & 1600 & 71 & 35.55 & 240 \\
\hline Intake & 1568.75 & 88.96 & 27.96 & 247.48 \\
\hline
\end{tabular}

\section{Care Considerations:-}

Regular nutritional visits are scheduled for one, two, three, six, nine and 12 months after surgery. Patients are placed on a clear liquid diet the day before surgery. Laboratory tests should be performed preoperatively and may be repeated in six months and annually thereafter. Patients may be discharged home on a bariatric full-liquid diet. Vitamin supplementation is required after surgery. A pureed diet is usually started after one week, and a soft diet, after four weeks.

\section{Justification:-}

Weight-loss surgery is the most effective treatment for morbid obesity, producing durable weight loss, improvement or remission of comorbid conditions, and longer life.

These techniques may further decrease the risk associated with bariatric surgery.

\section{Nutritional Requirement:-}

$\begin{array}{ll}\text { Energy } & -1600 \mathrm{kcal} \\ \text { Protein } & -71 \mathrm{gm} \\ \text { Fat } & -35.55 \mathrm{gm} \\ \text { Carbohydrate } & -240 \mathrm{gm}\end{array}$

Carbohydrate - $240 \mathrm{gm}$

Diet at hospital:-

\begin{tabular}{|l|l|l|l|}
\hline Date & Diet & Energy (kcal) & Protein $($ gm) \\
\hline $1^{\text {st }}$ day $(9 \mathrm{am})$ & NPO & - & - \\
\hline $1^{\text {st }}$ day $(3 \mathrm{pm})$ & Low salt soft diet & 1000 & 15 \\
\hline $2^{\text {nd }}$ day $(4 \mathrm{pm})$ & NPO & - & - \\
\hline $3^{\text {rd }}$ day $(11 \mathrm{am})$ & NPO & - & - \\
\hline $4^{\text {th }}$ day $(11 \mathrm{am})$ & NPO & - & - \\
\hline $4^{\text {th }}$ day $(2 \mathrm{pm})$ & Clear liquid diet $(20-30 \mathrm{ml} / \mathrm{hr})$ & 200 & - \\
\hline $5^{\text {th }}$ day $(11 \mathrm{am})$ & Clear liquid diet $(60-70 \mathrm{ml} / \mathrm{hr})$ & 400 & - \\
\hline
\end{tabular}




\section{Discharge Medication:-}

\begin{tabular}{|l|l|l|l|}
\hline MEDICINE & DOSE & FREQUENCY & PURPOSE \\
\hline Amlong & $5 \mathrm{mg}$ & $1-0-1$ & Anti- hypertension \\
\hline Olmesar & $40 \mathrm{mg}$ & $1-0-0$ & Helps to lower blood pressure \\
\hline Olmesar & $20 \mathrm{mg}$ & $0-0-1$ & Helps to lower blood pressure \\
\hline Cardivas & $25 \mathrm{mg}$ & $1-0-1$ & For treating high blood pressure \\
\hline Pantadoc & $40 \mathrm{mg}$ & $1-0-0$ & Antacid \\
\hline Calcium gummy bear & & $0-1-0$ & As a dietary supplement \\
\hline Vitamin gummy bear & & $0-0-1$ & As a dietary supplement \\
\hline
\end{tabular}

\section{Conclusion:-}

Patient is $41 \mathrm{yr}$ old man was admitted with above mentioned complaints. Endochronologist and Physician opinion was taken and advised followed. After all pre-operation evaluation; fitness and consent for the procedure, he underwent laparoscopic gastric sleeve resection on 06/09/13 under general anesthesia. Post operatively he was monitored in SICU and gradually shifted to the wards. He withstood the procedure well. Post operation hospital stay was uneventful. At the time of discharge vitals stable, surgical wound clear. Patient started clear liquid on third day after surgery which he was tolerating and discharged in stable condition.

Follow Up:-

\begin{tabular}{|r|r|r|r|}
\hline Timing & Meal & Menu & Amount \\
\hline $\mathbf{7 : 0 0}$ am & Early Morning & Coffee & $50 \mathrm{ml}$ \\
\hline $\mathbf{9 : 0 0}$ am & Breakfast & Coffee & $50 \mathrm{ml}$ \\
& & Dosa & $1 / 2$ \\
& & Sambar & $1 / 2$ cup \\
\hline $\mathbf{1 1 : 0 0 ~ a m ~}$ & Mid Morning & Tea & $50 \mathrm{ml}$ \\
\hline $\mathbf{1 : 3 0} \mathbf{~ p m}$ & Lunch & Rice & 1 cup \\
& & Fish Curry & 1 cup \\
& & Veg Salad & 1 cup \\
\hline $\mathbf{4 : 3 0 ~ p m}$ & Evening Snack & Tea & $100 \mathrm{ml}$ \\
\hline $\mathbf{9 : 0 0} \mathbf{~ p m}$ & Dinner & Chapathi & 4 Nos \\
& & Chicken Curry & 1 cup \\
\hline
\end{tabular}

\begin{tabular}{|r|r|r|r|r|}
\hline & Energy (Kcal) & Protein $\mathbf{( g m})$ & Fat $\mathbf{( g m})$ & CHO (gm) \\
\hline Requirements & 1600 & 71 & 35.55 & 240 \\
\hline Intake & 670.67 & 37.9 & 15.49 & 105.46 \\
\hline
\end{tabular}

\section{References:-}

1. Ogden CL, Carroll MD, Kit BK, Flegal KM. Prevalence of childhood and adult obesity in the United States, 2011-2012. JAMA. 2014; 311(8):806-814.

2. American Society for Bariatric and Metabolic Surgery. Impact of obesity. https://www.asmbs.org/patients/disease-of-obesity.

3. The practical guide, evaluation, and treatment of overweight and obesity in adults. National Institutes of Health. http://www.nhlbi.nih .gov/files/docs/guidelines/prctgd_c.pdf.

4. Bariatric surgery procedures. American Society of Metabolic and Bariatric Surgery. https://asmbs.org/patients/bariatric-surgery-procedures. 